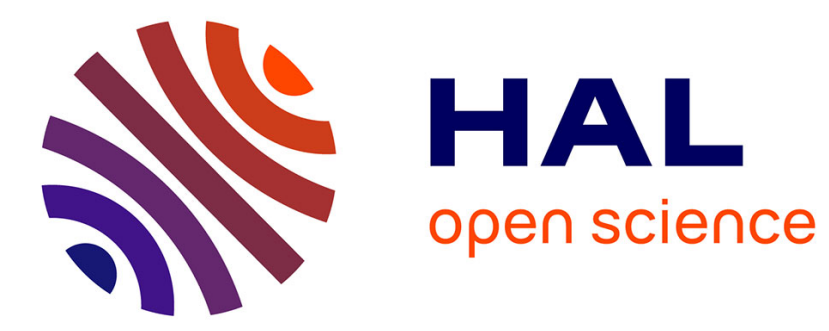

\title{
On the Voting Power of an Alliance and the Subsequent Power of its Members
}

Vincent Merlin, Marc Feix, Dominique Lepelley, Jean-Louis Rouet

\section{To cite this version:}

Vincent Merlin, Marc Feix, Dominique Lepelley, Jean-Louis Rouet. On the Voting Power of an Alliance and the Subsequent Power of its Members. Social Choice and Welfare, 2007, 28 (2), pp.181207. 10.1007/s00355-006-0171-6 . halshs-00010168

\section{HAL Id: halshs-00010168 https://shs.hal.science/halshs-00010168}

Submitted on 12 Apr 2006

HAL is a multi-disciplinary open access archive for the deposit and dissemination of scientific research documents, whether they are published or not. The documents may come from teaching and research institutions in France or abroad, or from public or private research centers.
L'archive ouverte pluridisciplinaire HAL, est destinée au dépôt et à la diffusion de documents scientifiques de niveau recherche, publiés ou non, émanant des établissements d'enseignement et de recherche français ou étrangers, des laboratoires publics ou privés. 


\title{
On the voting power of an alliance and the subsequent power of its members
}

\author{
Marc R. Feix ${ }^{*}$ Dominique Lepelley $†$ Vincent R. Merlin ${ }^{\ddagger}$ and Jean-Louis Rouet $\S$
}

November 2004

\begin{abstract}
Even, and in fact chiefly, if two or more players in a voting game have on a binary issue independent opinions, they may have interest to form a single voting alliance giving an average gain of influence for all of them. Here, assuming the usual independence of votes, we first study the alliance voting power and obtain new results in the so-called asymptotic limit for which the number of players is large enough and the alliance weight remains a small fraction of the total of the weights. Then, we propose to replace the voting game inside the alliance by a random game which allows new possibilities. The validity of the asymptotic limit and the possibility of new alliances are examined by considering the decision process in the Council of Ministers of the European Union.
\end{abstract}

\section{Introduction}

In a voting game, some players may have very similar interests and vote identically on most of the issues. In fact, in the extreme case where they agree on all issues, they form a de facto bloc and do not need at all to formalize any internal decision scheme. Here, as in Felsenthal and Machover [3], we study the case for which the coalition members have independent opinions on the different issues but agree to establish a decision rule which will give the alliance decision. Then, the alliance becomes a new player with a number of votes equal to the sum of its member's

*SUBATECH, Ecole des Mines de Nantes, La chanterie, 4 rue A Kastler, BP 20722, F-44307 Nantes cedex 3, France

${ }^{\dagger}$ CREM UMR6211 and CERESUR, Department of Economics, Université de La Réunion, 97715 Saint-Denis cedex 9, France

${ }^{\ddagger}$ Corresponding author. CREM UMR6211 and CNRS. MRSH bureau 230, Université de Caen, Esplanade de la Paix, 14032 Caen cedex, France

§Laboratoire de Mathématique, Applications et Physique Mathématique -UMR6628, Université d'Orléans, UFR des Sciences, F-45067 Orléans cedex 2, France 
votes; thus an alliance is defined as a bloc together with some internal decision rule ${ }^{1}$. This kind of alliance is not based on special affinity but simply takes advantages of the scale effects. An easy to understand example is given by the case of a five voters committee, deciding according to majority rule, each voter having one vote. If three voters ally, whatever the choice of the decision process inside the alliance is, this alliance will be a dictator and the power of a member belonging to the alliance will be the power of one among three instead of one among five. It can be checked that the Banhzaf-Penrose ${ }^{2}$ power index of an alliance member jumps from $3 / 8$ without alliance to $1 / 2$ with the alliance, while the members left out the alliance have now no power.

Remember that the power index describes a probabilistic concept. In some cases, the alliance brings a disadvantage to one of its members. In the previous example, a member of the alliance may have one opinion while the two others have the opposite one and the two exteriors to the alliance share his opinion. He must resist to the temptation of breaking the alliance since more often he will be one of the two members who win the vote against the three others (one in the alliance plus the two outside). These remarks outline the subtlety of the alliance concept based on the sole properties of a statistical power index.

In the present paper, we extend the work by Felsenthal and Machover in two directions. First, we analyze the relation between the voting power of a bloc and the voting powers of its members before the bloc has been formed, the power being understood as the ability to influence the outcome of the collective decision process. We know from [2] and [3] that 1) the Banzhaf voting power of a bloc of two voters $A$ and $B$ is equal to the sum of the powers of $A$ and $B ; 2$ ) for more than two members, the Banzhaf voting power of a bloc is not in general equal to the sum of the powers of its members. We obtain here new results for the so-called asymptotic limit for which the number of players is large enough and the bloc power remains a small fraction of the total power. Considering a large number of players in order to apply the central limit theorem is a well known strategy in game theory. This tradition dates back to Penrose: In his 1952 book, he stated (without any proof) that under these conditions, the power of a voter in a majority weighted game is proportional to his weight. This results is often referred to as the Penrose's law or the Penrose's limit theorem. Recently, Lindner and Machover [9] studied more precisely the conditions under which Penrose's result applies. The central limit theorem has also been used by Owen $[10,11]$ for the computation of the power in very large games using the multilinear extension of voting games. In a recent contribution, Leech [8] proposed a modified

\footnotetext{
${ }^{1}$ In this text, we stick to the terminological distinctions introduced by Felsentahl and Machover. When referring to a set of voters who combine into a single entity, always acting as a new single voter, we shall use the term 'bloc'. A 'coalition' is simply an arbitrary set of voters; it is not meant to imply that its members always vote in the same way. An 'alliance' is a bloc with an internal decision scheme, determining how the bloc will vote. Of course, the formation of a bloc changes the situation and creates a completely new game, as we have less players.

${ }^{2}$ Although the name of Banzhaf prevails in the literature to call his index of power [1], it is now well known that L.S. Penrose presented similar ideas about twenty years before (see $[12,13])$.
} 
version to give an estimate of the power in voting games which combine a small number of big players with a large number of small players in institutions such as the International Monetary Fund (182 players in 2002, with weights ranging from $0.01 \%$ of the mandates to $17.55 \%$ ) or the World Bank. Rather than using the model to give an estimate of the power, our objective is

just to use the properties that emerge in the asymptotic limit to get new general results on the power of blocs and their influences on the power of the other voters (like in Penrose's book). Our second objective is to study the design of the voting procedure inside the alliance. Clearly, an alliance will form only if allying is advantageous in term of voting power for each of its members. In [3] the alliance decision is taken by a qualified majority game and in some situations, there is no solution ensuring to each member a gain of power. Does it mean that this alliance cannot form? We will see that the answer to this question is negative: A new kind of game can be designed that makes the alliance possible.

The paper is organized as follows. Section 2 contains the general framework of our analysis and results concerning the voting power of a bloc of two members; The consequences of the formation of this bloc on the power of other voters are also analyzed. In Section 3, we consider the asymptotic limit case. The validity of the obtained results are examined by considering the decision process in the Council of Ministers of the European Union. In Section 4, the decision procedure inside the alliance is studied: We show that replacing the voting game suggested by Felsenthal and Machover [3] by a random game allows new possibilities. Some examples taken from the EU illustrate these possibilities. Section 5 summarizes the main conclusions of the paper.

\section{Framework and preliminary results}

\subsection{Voting game and voting power}

We consider a set of $N$ individuals (called voters or players) having to adopt or reject a proposal by means of voting. Once a proposal is submitted to the voters, each one will cast a vote. A vote configuration (or simply a configuration) lists the votes cast by the voters. We assume that the voters do not abstain: They vote either 'yes' or 'no'. Consequently, the number of possible configurations is $2^{N}$ when the number of voters is $N$.

A voting game specifies which configurations lead to the passage of the proposal (such configurations are said winning) and which ones lead to its rejection (losing configurations). We consider here the particular class of weighted voting games: Each voter is equipped with a weight and a configuration will be winning if the sum of the weights of the individuals voting 'yes' is at least equal to a required quota (denoted by $Q$ in what follows).

Given a configuration, a voter $A$ is said to be decisive if the voters other than $A$ are divided between 'yes' and 'no' in such a way that $A$ is in position to decide the outcome of the vote: 
If $A$ joins the 'yes' voters, then the proposal will be accepted, but if $A$ joins the 'no' voters, the proposal will be refused. In other words, the decision would have been reversed if $A$ has voted 'no' instead of 'yes' or 'yes' instead of 'no'. The Banzhaf power index of player A can be simply defined as the probability that $A$ is decisive. We suppose in this paper that voters act independently of one another and each votes 'yes' or 'no' with equal probability of $1 / 2$. Thus, denoting by $P(A)$ the Banzhaf power index of $A$ and by $\omega_{A}$ the number of configurations in which $A$ is decisive, we obtain ${ }^{3}$

$$
P(A)=\frac{\omega_{A}}{2^{N}}
$$

It is worth noticing that $P(A)$ can be given another interpretation. Let $X$ be the random variable that takes the value +1 if $A$ agrees with the outcome of the election and -1 if she/he disagrees. It can be proved that, under our probabilistic assumptions, we obtain $E[X]=P(A)$, where $E[X]$ is the expected value of $X$. Thus, $P(A)$ is also an index of correlation between $A$ 's preference and the collective preference. This interpretation, which can be traced back to Penrose [12] and Rae [14], can in some specific contexts facilitate the computation of the Banzhaf index (see Section 4).

\subsection{Power of a bloc of two voters}

We first analyze the voting power of a bloc of two voters, denoted by $A$ and $B$. Let $a$ and $b$ be the weights attached to players $A$ and $B$, respectively ${ }^{4}$. We assume first that $a>b$. The needed quota is $Q$ and $\bar{Q}$ is the number of 'yes' votes of a given configuration restricted to the $N-2$ voters excluding $A$ and $B$. If $\bar{Q} \geq Q$ or $\bar{Q}<Q-(a+b)$, then neither the bloc nor $a$ fortiori $A$ or $B$ can be decisive. In these two cases $\delta=Q-\bar{Q}$ is smaller than or equal to 0 or strictly larger than $a+b$ : The decision of the coalition is of no importance for the collective decision.

Now let us consider the case $0<\delta \leq a+b$. In this case, $\delta$ can be interpreted as the number of extra 'yes' votes needed by a configuration of the $N-2$ voters to reach the threshold $Q$. In order to compute the Banzhaf power indices $P(A)$ and $P(B)$ of $A$ and $B$ without bloc, as well as the Banzhaf power index $P(A B)$ of the bloc, we have to consider three cases gathered in tables 1a, 1b,1c. For $0<\delta \leq b, A$ is decisive two times and so is $B$. For $b<\delta \leq a, A$ is decisive four times while $B$ is never decisive ${ }^{5}$. For $a<\delta \leq a+b$, the situation is identical to the first one : $A$ and $B$ are decisive twice each. The bloc is always decisive for all these cases, that is, twice: If it votes 'yes', the collective choice will be 'yes', and if it casts a 'no' vote, the collective choice will be negative. Remembering that, with the bloc, we have one voter less, we

\footnotetext{
${ }^{3}$ What we call "Banzhaf power index" is what is sometimes called in the literature "absolute Banzhaf index" or "Banzhaf measure".

${ }^{4}$ Although in many real life examples the weights attached to the players are integers, we do not need this assumption in the paper.

${ }^{5}$ Notice that if $a=b$, this last case cannot happen; thus assuming $a>b$ does not reduce the generality of our results.
} 


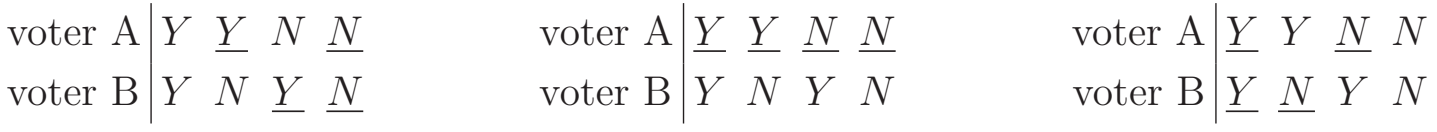

$$
\begin{aligned}
& \text { 1a: Case } 0<\delta \leq b . \quad \text { 1b: Case } b<\delta \leq a . \quad \text { 1c: Case } a<\delta \leq a+b \text {. }
\end{aligned}
$$

Table 1: Coalition of two players. The players in decisive situation are underlined.

have to multiply by two the numbers of configurations where the bloc $\mathrm{AB}$ is decisive in order to compare these numbers to the numbers of configurations where $A$ or $B$ are decisive (see equation (1)). Thus, we observe that the number of decisive situations of the bloc corresponds in each case to the sum of decisive situations for $A$ and $B$. Consequently, the power of a bloc of two voters is always equal to the sum of the powers of its members:

$$
P(A B)=P(A)+P(B) .
$$

Although relation (2) has already been proved in [2] for arbitrary simple games, the kind of reasoning we have just presented is used in the next section to analyze the influence of a bloc on the power of an external member. Next, we will build upon these results to obtain the main Proposition of Section 3.

\subsection{Power of a member external to a two voter bloc}

In addition to $A$ and $B$ who have $a$ and $b$ votes respectively and constitute the bloc, we focus on another voter $M$, who has $m$ votes. Is the power of $M$ modified by the existence of the bloc $A B$ ? The answer is yes. To continue with the introductory example, five voters with one vote each and a quota $Q=3$ have, without any bloc, a Banhzaf index power of $3 / 8$. If two ally, the bloc power is $3 / 4$, in accordance with relation (2), but the three voters left outside the bloc have their power decreasing from $3 / 8$ to $1 / 4$. Notice that, if the coalition goes from two to three voters, the power of this new bloc is $3 / 4+1 / 4=1$ indicating a dictatorship for the bloc. In order to evaluate the power of voter $M$ when the bloc $A B$ is formed, we have, as in the preceding sub-section, to examine all the cases where the other $N-3$ voters $(A, B, M$ excluded) contribute to $\bar{Q}$ 'yes' votes such that

$$
Q-(m+a+b) \leq \bar{Q}<Q
$$

Let $\delta=Q-\bar{Q}$ with $0<\delta \leq m+a+b$. Without loss of generality, we can take $a>b$, but we have to consider the five cases $m>a>b, m=a>b, a>m>b, a>m=b$ and $a>b>m$. Moreover, each case splits in three, since the largest of these three numbers can be larger, equal or smaller than the sum of the two others. We treat, as an example, the case $m>a>b$ with $m<a+b$. This leads to seven cases depending on the value of $\delta$ as illustrated Figure 1 . The decisive position of $M$ is examined when $\delta$ belongs to zone $I V$ of Figure $1(m<\delta \leq a+b)$ 
voter A $\mid \begin{array}{llllllll}Y & Y & Y & Y & N & N & N & N\end{array}$

voter B $\begin{array}{llllllll}Y & Y & N & N & Y & Y & N & N\end{array}$

voter M $\mathrm{Y} N \underline{Y} \underline{N} \underline{Y} \underline{N} \mathrm{Y} N$

\author{
Bloc AB $\begin{array}{llll}Y & Y & N\end{array}$ \\ voter $\mathrm{M} \mid \begin{array}{llll}Y & N & Y & N\end{array}$
}

Table 2: Case $m>a>b, m<a+b$ and for Table 3: Same as Table 2, but here a bloc zone $I V$ of Figure $1(m<\delta \leq a+b)$. Cases exists between $A$ and $B$. There is no decisive for which player $M$ is decisive are underlined position for $M$.

(no bloc between $A$ and $B$ ).

\begin{tabular}{r|ccccccc} 
Zones & I & II & III & IV & V & VI & VII \\
No bloc & 2 & 4 & 6 & 4 & 6 & 4 & 2 \\
Bloc & 2 & 2 & 2 & 0 & 2 & 2 & 2
\end{tabular}

Table 4: Number of cases for which player $M$ is decisive for each of the 7 zones of Figure 1.

under the two hypothesis: No bloc (Table 2) or bloc $A B$ (Table 3). Table 4 gives, for the seven possible positions of $\delta$, the number of times $M$ is decisive in the two cases. Taking into account the already discussed factor two of equation (1), we see that equality is not obtained for each zone. For example, in zone I, player M sees his Banzhaf power increase, as he is decisive in the same number of situations while the number of possible configurations has been divided by two. On the other hand, in zone IV, his Banzhaf power vanishes to zero. Thus, it is impossible to give any general statement about the evolution of $P(M)$ when $\mathrm{A}$ and $\mathrm{B}$ ally. Hence we also conclude that the bloc of $A$ and $B$ can modify the power of $M$, and this conclusion implies that the power of a bloc of three voters is not in general equal to the sum of the powers of its members.

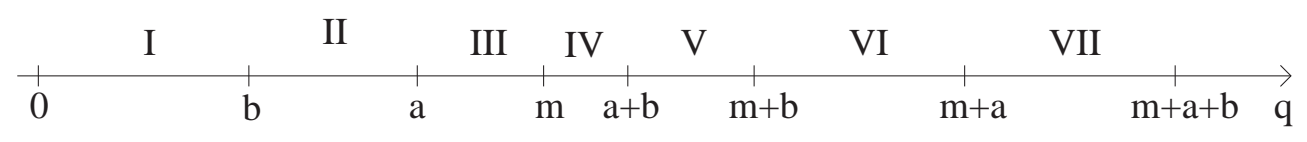

Figure 1: The impact of the AB bloc on the power of $\mathrm{M}$ when $m>a>b$ and $m<a+b$ : The seven different cases for the deficit of 'yes' votes.

\title{
3 Power of a bloc in the asymptotic limit
}

\subsection{The asymptotic limit model}

It is very difficult to find general properties when the bloc has more than two members. Nevertheless, in many fields, as statistical physics for example [6], things simplify and results can be obtained when $N$ (number of particles, stars, voters ...) goes to infinity. Using a concept borrowed from statistical physics [7], we consider a small interval $\Delta q$ and define a density $n(q)$ such that $n(q) \Delta q$ is the number of configurations for which the number of 'yes' votes belongs to the interval $[q, q+\Delta q]$. Stated in other terms, $n(q)$ is the mean probability density of the 
random variable $q$ in a sufficiently small interval $[q, q+\Delta q]$. The treatment is based on the central limit theorem [5] which states that if we consider $N$ independent random variables $x_{i}$ $(i=1, \ldots N)$, where $\left\langle x_{i}\right\rangle=m_{i}$ and $\left\langle\left(x_{i}-m_{i}\right)^{2}\right\rangle=\sigma_{i}^{2}$ (the bracket designing an ensemble average for each random variable $x_{i}$ ), the limit when $N \rightarrow \infty$ of the random variable $n(x)$, where $x=\sum_{i=1}^{N} x_{i}$, follows the distribution

$$
n(x) \sim \frac{1}{\sqrt{2 \pi} \sigma} \exp \left(-\frac{(x-m)^{2}}{2 \sigma^{2}}\right)
$$

where $m=\sum_{i=1}^{N} m_{i}$ and $\sigma^{2}=\sum_{i=1}^{N} \sigma_{i}^{2}$.

In the $N$ voter case, we denote by $q$ the total number of 'yes' votes (or mandates), and the random variable $x_{i}$ takes only two values 0 or $a_{i}$ with probability $1 / 2$. Then

$$
m=\frac{1}{2} \sum_{i=1}^{N} a_{i} \quad \text { and } \quad \sigma^{2}=\frac{1}{4} \sum_{i=1}^{N} a_{i}^{2} .
$$

The number of possible values for $q$ is at most $2^{N}$ and at least $N+1$ (all mandates are then equal). To approximate the vote configuration points by a continuous curve is a tricky problem: the interval $[q, q+\Delta q]$ must be sufficiently large to contain a big number of points (which will define the value of $n(q)$ for this interval) but sufficiently small to have low variation of $n(q)$ along the intervals.

This intrusion of probabilistic treatment in problems which have a deterministic solution (like the power of members of a qualified majority vote) brings three types of questions :

a - theoretical ones as: "under what circumstances this asymptotic treatment is valid ?"

b - practical ones as: "how big must be $N$ ?"

c - empirical ones as : "is this treatment valid for the EU with 9, 12, ., 27 members?"

In the Section 3.2, we give an a posteriori empirical justification for the use of this treatment, by comparing the normal approximation to the real curves of $n(q)$ obtained for EU27. But we agree that point (a) brings a lot of questions about its validity. Indeed $n(x)$ is defined as the ratio $\Delta p / \Delta x$, where $\Delta p$ is the number of points (here, vote configurations) in the interval $\Delta x$. The validity of (4) is entirely based on the decoupling between two scales: The number of points in the interval must be large enough compared to the interval, while the interval must remain relatively small. Statistic physics is entirely based on this double description: A microscopic world (here the $2^{N}$ configurations) and a macroscopic one captured by $n(x)$ with a scale of change much larger than the inter-particules (here, inter-configurations) distance. Although in physics the decoupling is much stronger because of the huge number of atoms or stars, we will see in section 3.2 that it is not bad for the EU27 example.

However, more can be said about the general conditions under which the approximation (4) is valid. Typically: 
- $2^{N}$ must be of course very large. The characteristic variation scale of $n(x)$ is given by $\sigma$. The obtention of the asymptotic limit is directly connected to the possibility of approximating $n(x)$ at point $x$ on an interval of order $a_{i}$ by the tangent of the righthand side of (4). Expanding $n(x)$ given by equation (4), one obtains

$$
n(x) \sim n\left(x_{0}\right)\left(1-\frac{\left(x-x_{0}\right)\left(x_{0}-m\right)}{\sigma^{2}}+\frac{1}{2}\left(x-x_{0}\right)^{2} \frac{\left(x_{0}-m\right)^{2}-\sigma^{2}}{\sigma^{4}}+\ldots\right)
$$

Because $x-x_{0}$ is of order $a_{i}$, while $x_{0}-m$ must be of order $\sigma$ for equation (4) to be valid, the second term in the parenthesis of equation (5) is of order $\left(a_{i} / \sigma\right)$ and is taken into account in the following asymptotic computation. The third term of order $\left(a_{i} / \sigma\right)^{2}$ is neglected.

- The values of $m_{i}$ and $\sigma_{i}$ must be completely negligible compared to $m$ and $\sigma$ respectively for the approximation to be valid. This implies that, in the EU case, difficulties will appear if the mandates $a_{i}$ of state $i$ are taken proportional to the population, because of the large ratio of population between Malta (0.38 millions inhabitants) and Germany (82.0 millions). For example, the validity of the approximation by (4) will be better in the case of weights proportional to the square root of the population.

- The weights must be sufficiently scattered, so that all the $2^{N}$ configurations give different sums of weights. The assumption that the weights are sufficiently scattered ensures that we avoid pathological cases (see for example the introductory example in Lindner and Machover [9], where all the voters except one have the same weight) and leads to a better approximation. To have the full set of $2^{N}$ different points a practical rule could be to give to each country a number of mandates with as many as possible significant figures. If the weights are integers we will get at most $2 m+1$ values for $x$. But again, if the weights in the European Union are proportional to the square root of the population of a country, we are quite sure that the $2^{N}$ configurations will all lead to different sums of 'yes' vote.

- The relative quota must be close to $m$. The difficulty is the validity of the tail of the Gaussian distribution. As already mentioned, the central limit theorem imply that $\left(x_{0}-\right.$ $m) / \sigma$ is smaller than a finite value. More precisely, its means that for a given $\alpha$ one can find $N_{0}$ such that for $N>N_{0}$, the approximation $n(x)$ is valid up to $\alpha \sigma$. Recently, some results have been obtained for the values around the end points $x=0$ and $x=\sum_{i=1}^{N} a_{i}$ [15]. Notice that if the number of states goes to infinity, the Gaussian is more and more peaked around $m$ and both probability of approval and the powers of all states go to zero.

Finally, the case of $N$ voters with one mandate each deserves a special mention. This fully degenerated case (all $a_{i}$ equal) give a distribution function $n(x)$ which is a sum of Dirac distributions which envelope is well fitted by the Gaussian. To recover a smoothed curve for $n(x)$ we must take average on an interval $\Delta x>>1$. Of course, this case with one mandate each can be easily handled. But if we add a few number of voters with a small weight each (for example 3 players with weight 0.1 each) we cannot ignore any more the fundamentally discrete nature of the $x$ variable and a more "microscopic" description is needed. 
In conclusion, although the approach will be valid in most of the cases when the conditions we have described are fulfilled, we must be aware that pathological cases exist. We agree that more work is needed on these questions (see for example the recent results obtained by [9]) but we claim that the description of large sum of discrete random variables through the density distribution $n(x)$ is a fundamental tool to obtain quantitative informations, as we will see in the next sections.

\subsection{Application to the European Union.}

To give some examples on the range of application of this approximation, we discuss the different shapes of the real curves $n(q)$ and their normal approximations for the decisions schemes in the council of ministers of the European Union from 1973 (displayed on Figures 2, 3 and 4) till the Treaty of Nice.
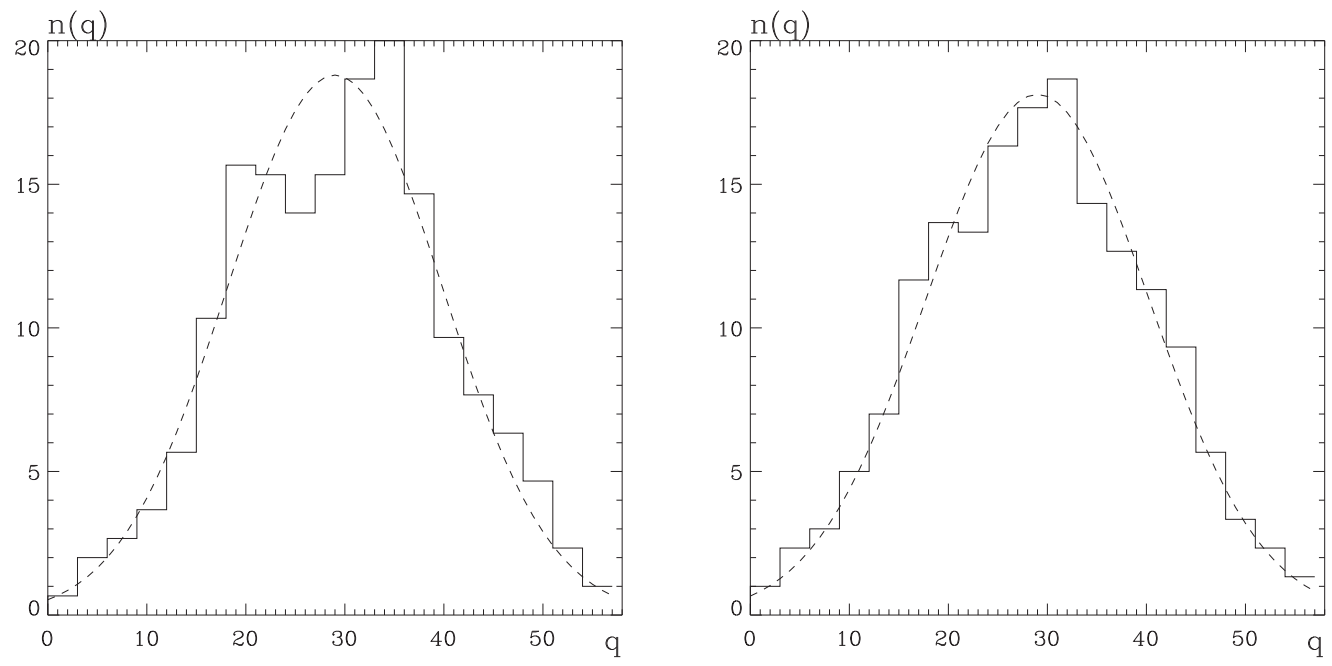

Figure 2: $\mathrm{n}(\mathrm{q})$ and its normal approximation for EU9, a. The mandates are those allotted to each of the nine countries, $10,10,10,10,5,5,3,3,2$. b. The number of mandates is proportional to the square root of the population, the sum is normalized to $58: 12.1,10.3$, $10.3,10.2,4.3,4.3,3.1,2.6,0.9$.

In 1973, France, Germany, Italy and United Kingdom were represented by 10 mandates each, Belgium and Netherland by 5 mandates each, Ireland and Denmark by 3, and, at last, Luxembourg by only two mandates, for a total of 58 . Figure 2 a reads as follows : $\Delta(q)=3$ and $n(q)$ gives the number of coalitions which gather in between $q$ and $q+\Delta(q)$ (excluded) votes divided by $\Delta(q)$. For example, the number of coalitions which gather 3 , 4, or 5 'yes' votes is 6 , which gives a value of 2 for $n(q)$. As we can see on figure $2 \mathrm{a}$, the normal approximation through the normal law is not good. This is partly due to the fact that the mandates are not sufficiently scattered. If, on the contrary, we take mandates proportional to the square roots 
of the populations of the member states $^{6}$, we get a more scattered dispersion of the $q_{i}$ and the approximation is better. This illustrates the previous remark on the benefit of keeping a sufficient number of figures for the mandates number $a_{i}$.
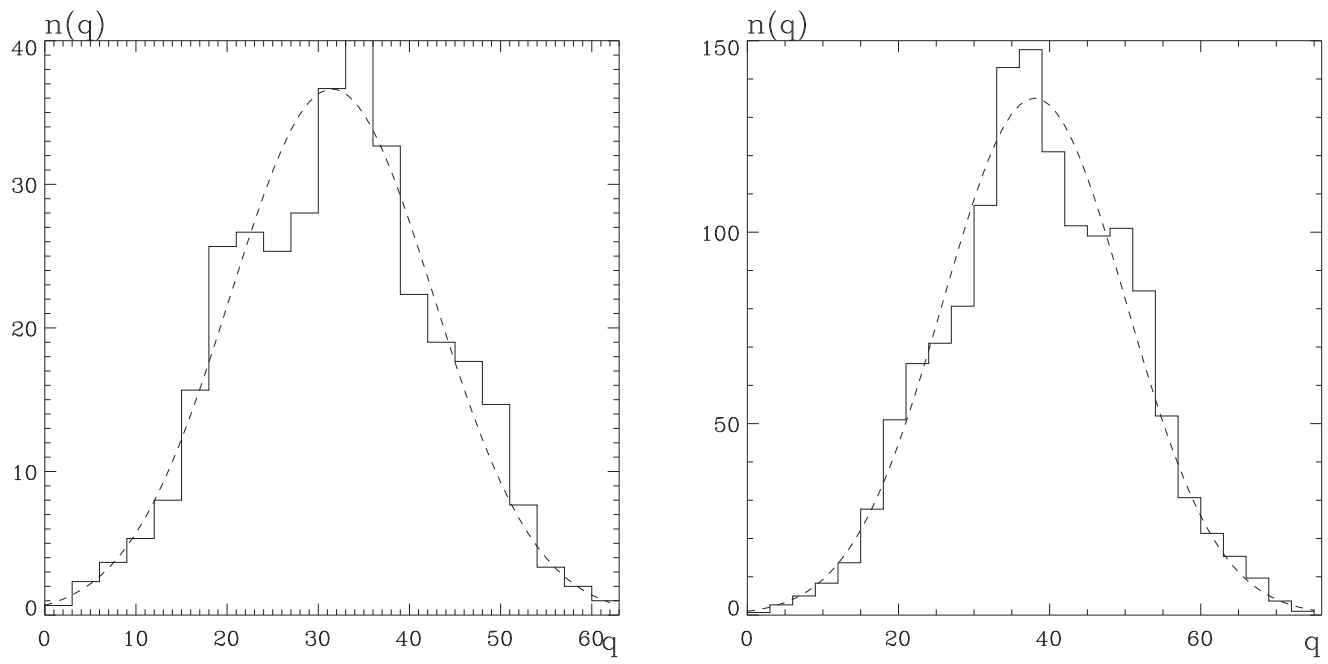

Figure 3: n(q) and its normal approximation for EU10 and EU12, with $\Delta(q)=3$. The mandates of the states are those attributed for EU10 and EU12 respectively.
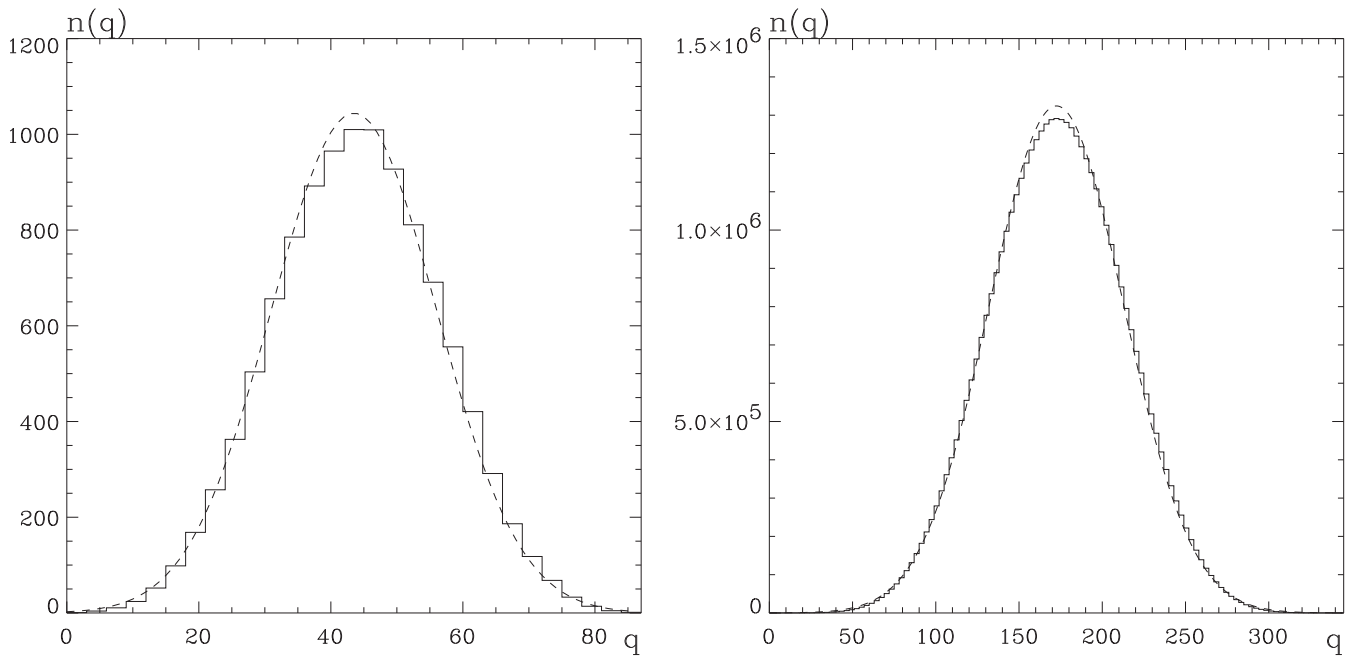

Figure 4: $\mathrm{n}(\mathrm{q})$ and its normal approximation for EU15 and EU27, with $\Delta(q)=3$. The mandates of the states are those attributed for EU15 and EU27 respectively.

Indeed, the central limit approximation becomes more pertinent when the number of states increases. Though the approximation is still not very convincing for EU10 and EU12 (see Figure 3 ), it is already quite good for EU15, and becomes very close to the reality for EU27, as seen

\footnotetext{
${ }^{6}$ The population of the different countries in EU27 and their respective square roots are displayed on Table 3 in Felsenthal and Machover [3].
} 
on Figure 4 and even better with the square root law for $a_{i}$, as shown Figures 5, 6, 7 .

Since the 2004 enlargement, the European Union is thus an important case where the smoothed character of $n(q)$ has been established, and where we can use the asymptotic approach to state new properties on the power of a player external to a bloc.

\subsection{The power of player $M$ in the asymptotic limit}

In this continuous limit, we will compute the Banhzaf index of voter $M$, with and without the bloc $A B$ for the case already studied section 2.3 and summarized Table 4.

In the asymptotic limit, the distribution of the 'yes' votes from the $N-3$ other voters is given by a new density function $n(q)$, according to equation (4). Expanding $n(q)$ up to first order (terms in $N^{-1 / 2}$ but ignoring second derivative of order $N^{-1}$ ) and bringing the origin of $q$ at the mid point $(m+a+b) / 2$, we write

$$
\begin{aligned}
n(\Delta q) & =n(q-(m+a+b) / 2)+n^{\prime}(q-(m+a+b) / 2) \Delta q \\
& =n_{0}-K \Delta q
\end{aligned}
$$

where

$$
n_{0}=n(q-(m+a+b) / 2) \quad K=n^{\prime}(q-(m+a+b) / 2) \quad \Delta q=q-\frac{m+a+b}{2}
$$

The number of times voter $\mathrm{M}$ is decisive with no bloc is obtained by the computation of the integral of $n_{0}-K \Delta q$ on the different zones (I to VII) of Figure 1 weighted by the corresponding coefficients given Table 4. Now, noticing from Table 4 that the number of decisive positions is an even function of $\Delta q$, we see that the contribution of $K \Delta q$ will vanish. We must compare

$$
\begin{gathered}
2 \int_{0}^{b} n_{0} d \Delta q+4 \int_{b}^{a} n_{0} d \Delta q+6 \int_{a}^{m} n_{0} d \Delta q+4 \int_{m}^{a+b} n_{0} d \Delta q+6 \int_{a+b}^{m+b} n_{0} d \Delta q+4 \int_{m+b}^{m+a} n_{0} d \Delta q+2 \int_{m+a}^{m+a+b} n_{0} d \Delta q \\
\quad=[2 b+4(a-b)+6(m-a)+4(a+b-m)+6(m-a)+4(a-b)+2 b] n_{0}=8 m n_{0}
\end{gathered}
$$

(case for which there is no bloc), to

$$
[2 b+2(a-b)+2(m-a)+0(a+b-m)+2(m-a)+2(a-b)+2 b] n_{0}=4 m n_{0},
$$

(case for which the bloc exists).

Now, taking into account the normalization factor two for equation (10), we see that we have equality between equation (9) and (10). It is easily (but tediously) checked that the other cases corresponding to the other inequality between $a, b$ and $m$ give the same result. Consequently, the following result can be stated : 
Proposition 1 When the asymptotic limit approach is valid, the power of a member external to a bloc of two members is not modified by its formation.

Now, since, in the asymptotic limit, the power of an external member is not modified, and remembering that the power of a two member bloc is the sum of the power of its members, we can built a multi-voter bloc aggregating the countries one by one and can state :

Proposition 2 When the asymptotic limit approach is valid, the power of a bloc is equal to the sum of the powers of its members.

Of course when the bloc becomes big, we are no longer in the asymptotic limit. The points are no longer scattered enough along the $q$ axis, and the approximation by the normal law may fail. The number of votes of the bloc must always remain negligible compared to the total number of votes.

We now illustrate our analysis by considering the decision process in the EU27. We have studied two types of bloc: The biggest states and the smallest. The result are summarized Table 5 and 6. We see that, for the bloc of the smallest states (Table 6), the asymptotic limit is valid, while for the bloc of the three biggest ones (Germany, U.K., France; Table 5), the bloc power is $18 \%$ larger. But this result is not surprising since with 87 votes, this bloc has more than $25 \%$ of the total number of votes.

\begin{tabular}{cccc}
$N$ & Bloc & Sum of the powers & Bloc power \\
\hline 1 & Germany alone & 0,032688 & 0,0327 \\
2 & Germany and UK & 0,065376 & 0,0654 \\
3 & Germany, UK and France & 0,098064 & 0,1162
\end{tabular}

Table 5: Power for a bloc of big countries.

\begin{tabular}{lccc}
$N$ & Bloc & Sum of the powers & Bloc power \\
\hline 1 & Malta alone & 0,003958 & 0,0040 \\
2 & Malta and Luxembourg & 0,009209 & 0,0092 \\
3 & Malta, Luxembourg and Cyprus & 0,014460 & 0,0146 \\
4 & Malta, Luxembourg, Cyprus and Estonia & 0,019711 & 0,0198
\end{tabular}

Table 6: Power for a bloc of small countries.

\subsection{Relation power-mandates in the asymptotic limit}

Beside the results given in the bloc problem studied in section 2.3, another important relation, obtained in the asymptotic limit, is the proportionality between the power and the weights. 
This fact has been suggested first by Penrose [13] which states that in the majority game (the quota is exactly $\left.m=(1 / 2) \sum_{i=1}^{N} a_{i}\right)$ :

$$
P(A) \approx a \sqrt{\frac{2}{\pi \sum_{i=1}^{N} a_{i}^{2}}}
$$

by using a central limit argument for a large number of players. Since that times, the Penrose law is known to be generally valid, but precise proofs only exist in some particular cases (see in particular Lindner and Machover [9]). We propose here to look at this result in a different way, which does not use a priori a specified value for the quota. To show that the Penrose limit theorem holds, we consider $N$ states plus 2 states $A$ and $B$, which have respectively $a$ and $b$ mandates. The $N$ other states give $2^{N}$ configurations, each represented by a point on the $q$ axis. Around the quota $Q$ (taken as the origin) we can write, in the asymptotic limit, the following first order relation for the equation (4) of Section 3.1.

$$
n(\Delta q)=n_{0}-K \Delta q .
$$

Now, if $B$ votes 'no', $A$ is decisive for all the configurations between $-a$ and 0 and if $B$ votes 'yes', $A$ is decisive for all configurations between $-a-b$ and $-b$ (see Table 1 ). To obtain the voting power of $\mathrm{A}$, we must compute

$$
\frac{1}{2} \int_{-a}^{0}\left(n_{0}-K \Delta q\right) d \Delta q+\frac{1}{2} \int_{-a-b}^{-b}\left(n_{0}-K \Delta q\right) d \Delta q=a\left[n_{0}+\frac{K}{2}(a+b)\right]
$$

The same computation for the power of $B$ gives $b\left[n_{0}+\frac{K}{2}(a+b)\right]$ and consequently, up to first order, the powers are proportional to the numbers of mandates:

$$
\frac{P(A)}{P(B)}=\frac{a\left[n_{0}+\frac{K}{2}(a+b)\right]}{b\left[n_{0}+\frac{K}{2}(a+b)\right]}=\frac{a}{b}
$$

Remembering that, in the asymptotic limit, $n(q)$ is given by the central limit theorem with a scale going as $N^{1 / 2}$, the first derivative is of order $N^{-1 / 2}$ and the second one of order $N^{-1}$. Consequently, the above result implies a possible deviation of order $N^{-1}$. It is important to compare the power of $B$ and $A$ to deal with the same set of configurations, that is to deal with the same $n_{0}$ and $K$. Then we show that the proportionality to $a$ and $b$ of $P(A)$ and $P(B)$ is correct up to first order included. This demonstration is not possible if we treat separately $\mathrm{A}$ and $\mathrm{B}$ with a set of configurations which include $\mathrm{B}$ and vice-versa. If we treat separately $\mathrm{A}$ and $\mathrm{B}$, this demonstration is only valid up to zero order because, for the computation of A, the set of configurations includes B and for B it includes A. Consequently, the $n_{0}$ and $K$

are not exactly the same. Notice at last that we never specified the value of the quota in the demonstration. Of course, the whole argument we have developed in this section is only valid if the approximation of $n(x)$ by (4) is good enough.

\subsection{The validity of Penrose's limit theorem in EU27}

Let us check this relation power-mandates in the EU 27 case with two examples. First, to avoid pathological cases which can occur with small integer votes, we illustrate the use of the normal 
approximation with mandates proportional to the square root of the population in EU27 and a quota of $60 \%$ of the total number of votes, as in Felsenthal and Machover [4] (that is $Q=207$ ). The column of Table 7 in [4] labelled "quotient" gives the ratio Banhzaf index by Pop ${ }^{1 / 2}$, where $P o p$ is the population of the member state and is given to compare the power of a citizen in the 27 countries. But, since the number of votes is proportional to $\operatorname{Pop}^{1 / 2}$ (with a very small round off error; 3 significant figures are kept), it is also a measure of the proportionality powervotes. The agreement is excellent (variation of order .5\%) and much better than the value we expected. The reason is the following. If we normalize the total number of votes to 345 (as the authors do), the center of the Gaussian is at 172.5 and the square root of the variance is $\sigma=39.84$. But, for the value $x=\sigma$ of the centered Gaussian reading

$$
f(x)=\frac{1}{\sqrt{2 \pi}} \frac{1}{\sigma} \exp \left(-\frac{x^{2}}{2 \sigma^{2}}\right),
$$

the second derivative cancels giving here a value of

$$
q=172.5+40=212.5
$$

For this value of $q$, the quota of $61.5 \%$, is very closed to the $60 \%$, value selected by Felsenthal and Machover to compute the power. Figure 5 gives, for this $P o p^{1 / 2}$ model, the comparison between the asymptotic (Gaussian) model and the real $n(q)$ obtained with the 27 voters. The agreement is quite good and we clearly see the linear zone around $q=205$ (see Figure 6). Around the value 255 (the value of the quota decided in the Nice Treaty for the 27 members), we can expect a not so good agreement since the second derivative certainly plays a role (see Figure 7). However, notice that in these cases, its is still possible to use reasonably a linear approximation although we are far away from the quota $m$.

Finally, we must stress that the Gaussian approximation (with its $N^{1 / 2}$ scale) is only valid for a deviation $x$ not increasing faster than $\alpha N^{1 / 2}$ where $\alpha$ is a finite number. Consequently, for very large $N$, it does not apply for $x$ increasing as $N$. When $N \rightarrow \infty$, the quota must tend to $50 \%$. Indeed, keeping the quota at its actual value and increasing $N$ gives a probability of an approval vote quickly decreasing (it is already $2 \%$ with the actual qualified majority) and all state power tends to 0 .

With the values given by the Nice treaty, although neither $N$ is very large nor the quota closed to $50 \%$ (with moreover a quite irregular and high degenerate distribution votes), we are not very far from the asymptotic limit. Table 7 shows a fair proportionality votes-power (the power value have been taken from the Table 9 of [4]). The agreement is good except for big countries (27 or 29 votes) where it is only fair. In such cases an interval of 27 or 29 is not small enough to neglect the deviation from the linear approximation of $n(q)$. Another problem is due to the fact that the number of votes have been rounded to not very large integers and also that many states have the same number of votes. This situation is reminiscent of some degenerate quantum systems where, indeed, large fluctuations do occur. When these effects are absent and with a quota $(60 \%)$ closer to $50 \%$ we have the excellent agreement discussed above. 


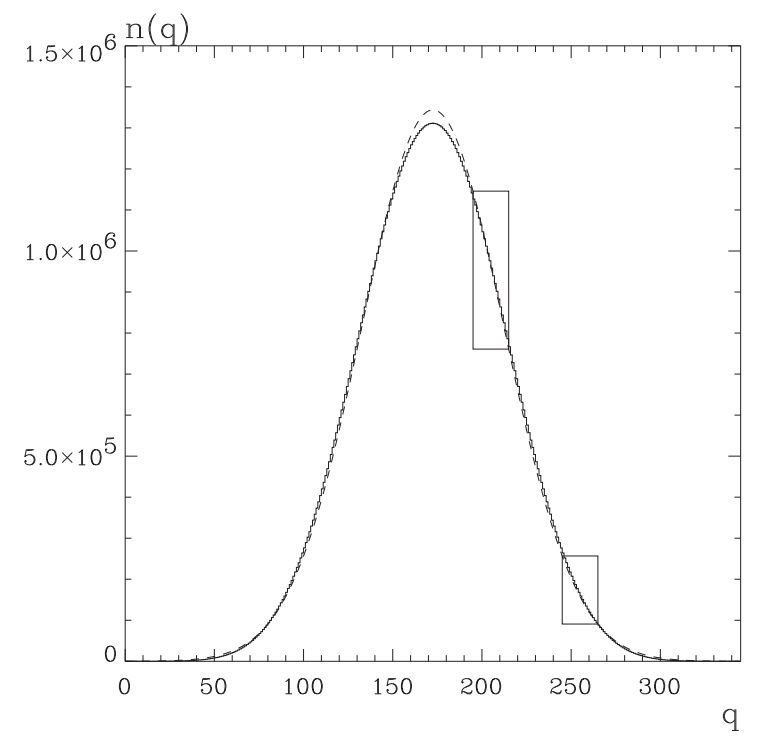

Figure 5: $\mathrm{n}(\mathrm{q})$ and its normal approximation for EU27, with $\Delta(q)=1$. The mandates of the states are taken in accordance with the square root of the population
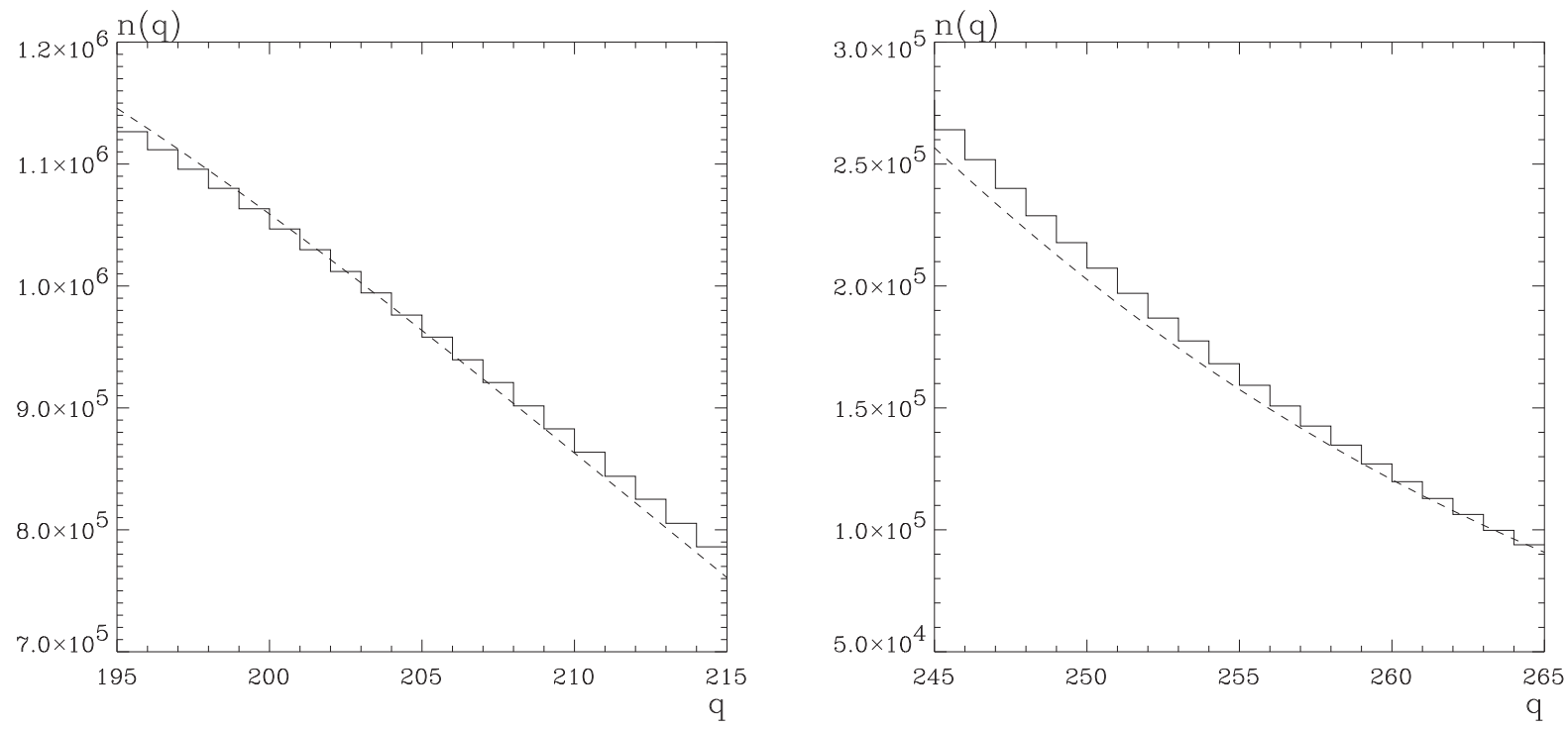

Figure 6: A zoom around 205 for $\mathrm{n}(\mathrm{q})$ and Figure 7: A zoom around 255 for $\mathrm{n}(\mathrm{q})$ and its normal approximation for EU27, with its normal approximation for EU27, with $\Delta(q)=1$ $\Delta(q)=1$

To continue on the applications of the normal approximation in the asymptotic case, we try to valuate the power of Malta in EU27 in this way. We only consider the first game with a quota $Q=258$ (without the conditions on the number of states and a threshold for the population ${ }^{7}$ ). Malta gets $\Delta q=3$ mandates, and the other 26 countries gather a total of 342 votes. So, the

${ }^{7}$ For more details on the different games and their variants, see Felsenthal and Machover [3]. We here focus on the game $\mathcal{W}_{27}$ described on pages 440-441. 


\begin{tabular}{lc} 
Number of votes & Power/number of votes $\times 10^{3}$ \\
\hline 29 & 1.127 \\
27 & 1.154 \\
14 & 1.278 \\
13 & 1.284 \\
12 & 1.289 \\
10 & 1.298 \\
7 & 1.309 \\
4 & 1.313 \\
3 & 1.319
\end{tabular}

Table 7: Number of votes and ratio of the power over the number of votes for countries of U.E. 27

mean is 171 , and the variance $\sigma=\sqrt{1633}=40.41$. The distribution of $q_{i}$ is thus given by:

$$
n(q)=2^{26} \frac{1}{\sqrt{2 \pi}} \frac{1}{\sigma} e^{-\frac{(q-171)^{2}}{2 \sigma^{2}}}
$$

The Banhzaf power index is evaluated by the integral

$$
\frac{1}{2^{26}} \int_{0}^{\Delta Q} n(258-\epsilon) d \epsilon
$$

which gives the number of times the 3 'yes' votes of Malta allows to pass the quota. An approximation of this integral is given by a Taylor expansion of $n(q)$ at second order (for example)

$$
\int_{0}^{\Delta q} n(258-\epsilon) d \epsilon \sim n(258) \Delta q-n^{\prime}(258) \Delta q^{2} / 2+n^{\prime \prime}(258) \Delta q^{3} / 6
$$

with

$$
n(258-\epsilon)=n(258)-n^{\prime}(258) \epsilon+n^{\prime \prime}(258) \epsilon^{2} / 2
$$

Thus we obtain the following figures:

\begin{tabular}{lr}
\hline second order & 0.00316071 \\
using the integral & 0.00316088 \\
\hline real value & 0.00337
\end{tabular}

We can notice that the second order is a good approximation of the integral and that the asymptotic model is correct (the deviation is less than $6.5 \%$ ). Similar observation can be made for the bigger countries: The estimation for the Germany using the integral is 0.02596 , for a real value equal to 0.02736 .

Now, we can perform the same computations with the rule suggested by Felsenthal and Machover: Each state has a number of mandates proportional to the square root of its population, and the quota is $Q=0.6(345)=207$. The number of mandates for Malta is $\Delta q=2.2, \sigma=39.83$ and the mean value $m=171.45$. Following the same treatment, we get 
\begin{tabular}{lr}
\hline asymptotic model & 0.0151585 \\
\hline real value & 0.015558
\end{tabular}

with a deviation less than $3 \%$. This second model gives a better agreement because the $\Delta q$ is lower and also because the ratio $(Q-m) / \sigma$ passes from 2 to 0.9 . We are consequently more in the center of the gaussian and leave the edges for which the continuous approximation model is no more correct. Similarly, the integral estimation gives a power of 0.22854 for Germany, while the real value is 0.23175 .

\section{Decision and Power inside the alliance}

\subsection{Voting game inside the alliance}

The strength of an alliance comes from the fact that its members always vote as a bloc. If everybody agrees to vote either 'yes' or 'no', then there is no problem. But in the (frequent) case of a disagreement between its members (recall that the preferences of the alliance members are independent), the alliance must design a rule to decide what will be its vote. This rule will not only give the alliance vote but also the power, inside the alliance, of each of its member. The first idea that comes in mind (and that is used in [3]) is to play a voting game inside the alliance.

We will call $P(A)$ the power of $A$ when there is no alliance, $P$ the alliance power and $p(A)$ the power of $A$ inside the alliance. The final power $\tilde{P}(A)$ of $A$ when the alliance exists is

$$
\tilde{P}(A)=p(A) P \text {. }
$$

This relation is given in [3] (Theorem 4.1) and directly follows from the independence assumption that underlies our model. Of course, the alliance will form only if $\tilde{P}(A)$ is larger than (or equal to) $P(A)$ for all alliance members. The problem is to design the voting game inside the alliance (weights of each of its members and quota) in such a way that this condition is fulfilled.

Let see first some examples. We begin with a two member alliance. Let us call $\tilde{a}$ and $\tilde{b}$ the number of votes of $A$ and $B$ inside the alliance and $\tilde{Q}$ the quota (we use quantities with a tilde to avoid confusion with the original game). Let us suppose that $P(A)>P(B)$. This assumption leads to take $\tilde{a}>\tilde{b}$ and three cases are possible for $\tilde{Q}$ :

1. $\tilde{Q}>\tilde{a}$; then $p(A)=p(B)=1 / 2$, giving $\tilde{P}(A)=(P(A)+P(B)) / 2=\tilde{P}(B)$ and $A$ will refuse the alliance,

2. $\tilde{a}>\tilde{Q}>\tilde{b}$ will make $A$ a dictator, a situation refused, of course, by $B$,

3. $\tilde{b}>\tilde{Q}$ brings back the first situation. 
Thus, using a voting game as a decision procedure makes a two member alliance impossible in the (general) case where $P(A)>P(B) .^{8}$

Consider now a three member alliance. First we suppose three voters with an equal number of votes and, consequently, an identical power taken equal to 1. Let us assume that the alliance power is the sum of its member powers, i.e. $P=3$. Symmetry imposes that, in the alliance voting game, we take $\tilde{a}=\tilde{b}=\tilde{c}=1$ and $\tilde{Q}=3 / 2$. Then $p(A)=p(B)=p(C)=1 / 2$ and each member sees its power jumping from 1 to $3 / 2$ and the alliance is approved. Now, let us suppose that, in the absence of any alliance, the powers are respectively proportional to 5,3 and 1 for $A, B$ and $C$, with again $P$ equal to the sum of the member's powers, i.e. $P=9$. Supposing $\tilde{a}>\tilde{b}>\tilde{c}$, the reader will examine the two cases $\tilde{a}<\tilde{b}+\tilde{c}$ or $\tilde{a}>\tilde{b}+\tilde{c}$. In each of these cases, seven choices of $\tilde{Q}$ are possible (see Appendix I). After examination of the $2 \times 7$ possible cases, it is easily seen that no case allow to increase simultaneously the power of the 3 players.

Moreover, a subtle point must be discussed. Consider again a three member alliance with initial powers proportional to $10,3,2$ and $P=15$. We examine again the $2 \times 7$ possible cases given in Appendix I and see that only the choice of quota in zones III or $\mathrm{V}$ will lead to an acceptable solution for the 3 players, with namely $\tilde{P}(A)=3 / 4, \tilde{P}(B)=\tilde{P}(C)=1 / 4$, then $\tilde{p}(A)=45 / 4$, $\tilde{p}(B)=\tilde{p}(C)=15 / 4$ and all players have their initial power increased by the alliance. Nevertheless the solutions in zone III or V imply a destruction of the symmetry between the 'yes' and 'no' vote of the alliance (5/8 or 3/8, see last line of Table 10 and 11$)$. A priori, this symmetry is needed to compute both the alliance power and the power of the members outside the alliance. Nevertheless, the examination of all the vote configurations, taking into account the probability $p$ and $q$ of 'yes' or 'no' vote of the alliance - the number outside the alliance keeping the 'yes' and 'no' symmetry - shows that the alliance power is unaffected by this dissymmetry, while the power of the numbers outside the alliance is modified and depends on the precise value of $p$ and $q$. Consequently, the absolute Banzhaf index is still given by formula (12), but the normalized index is modified. This raised the question of the meaning of the absolute and normalized Banhzaf indices. In particular, what is the index the voters seek to increase?

The symmetry breaking can be avoided by a trick which is a first step in the direction of the random game which we will introduce in the next section. We notice that a game with a quota $Q$ and a game with a quota $T-Q$ (where $T=2 m$ is the total number of votes of all the players) are just the same game where the roles of the 'yes' and 'no' have been switched. The powers of the different members are unchanged and the probabilities of 'yes' and 'no' at the collective level are just exchanged. The needed symmetry is then recovered in the following way:

1. select a $\tilde{Q}$ and $\tilde{a}, \tilde{b}, \tilde{c}, \ldots$ which provides a solution accepted by all the members,

2. select either $\tilde{Q}$ or $\tilde{T}-\tilde{Q}$ with equal chance $1 / 2$,

${ }^{8} \mathrm{~A}$ two member alliance is however possible when $P(A)=P(B)$, as noticed in [3] (Theorem 4.2). 
These considerations suggest that the introduction of a random drawing can restore a certain symmetry and allows a smoothed connection with the random game.

\subsection{The random game as a deciding model in the alliance}

Consider the case of a two member alliance $(A$ and $B)$. The situation is entirely described by the four cases: $A$ and $B$ vote 'yes'; $A$ votes 'yes', $B$ votes 'no'; $A$ votes 'no', $B$ votes 'yes'; $A$ and $B$ vote 'no'. In the first and last cases, deciding on the alliance vote is obvious since the two members agree. In the case where $A$ and $B$ disagree, we propose to pick up the $A$ decision as the alliance decision with a probability $x$ using a random game. How to select $x$, and first what are $p(A)$ and $p(B)$ in this new game? The simplest way to answer the last question is to use the correlation interpretation of the Banzhaf power index: we compute the average value of a variable equal to +1 if the decision of the considered member agrees with the global decision and -1 in the contrary case. Thus

$$
p(A)=\frac{1}{4}(1+x-(1-x)+x-(1-x)+1)=x
$$

and

$$
p(B)=\frac{1}{4}(1+(1-x)-x+(1-x)-x+1)=1-x .
$$

Notice that these results can also be obtained by using the regular interpretation. If, in the situation where $A$ and $B$ vote 'yes', $A$ changes its mind, we go from a certain answer 'yes' for the global position to a 'no' with a probability $x$; The contribution to the power of $A$ is consequently $x / 4$. Next, if $A$ changes its mind, starting from $A$ votes 'yes', $B$ votes 'no', we go with certainty to a global answer 'no', but only for a percentage $x$ of the starting situation. Again the contribution to $p(a)$ is $x / 4$. The two last situations bring $x / 4$ each, for a total power $p(A)=x$ and $p(B)=1-x$.

Now, from

$$
\tilde{P}(A)=x(P(A)+P(B))
$$

and

$$
\tilde{P}(B)=(1-x)(P(A)+P(B))
$$

and the two last relations

$$
\tilde{P}(A) \geq P(A), \quad \tilde{P}(B) \geq P(B),
$$

we deduce that

$$
x=\frac{P(A)}{P(A)+P(B)},
$$

and we finally obtain 


$$
\tilde{P}(A)=P(A) \quad \text { and } \quad \tilde{P}(B)=P(B) .
$$

The alliance is no longer impossible and leaves unchanged the power of its members.

We now consider the case of a three member alliance. In two configurations ( $A, B, C$ vote 'yes' and $A, B, C$ vote 'no') the decision is obvious. In the six other configurations, one member has an opinion which is not that of the two others. We call respectively $x, y, z$ the probabilities to follow the opinion of the isolated member if this one is respectively $A, B$ or $C$. For example if $A$ and $C$ vote 'no', $B$ votes 'yes', we call $y$ the probability that the alliance votes 'yes'. The symmetry between the 'yes' or 'no' decisions is automatically fulfilled since in the case where $A$ and $C$ vote 'yes' and $B$ votes 'no', $y$ is also the probability that the alliance votes 'no'. Now, using once again the correlation interpretation of the power indices, we can easily derive $p(A)$, $p(B)$ and $p(C)$, the powers inside the alliance ${ }^{9}$. A little algebra gives

$$
\begin{aligned}
& p(A)=\frac{1}{2}(1+x-y-z), \\
& p(B)=\frac{1}{2}(1-x+y-z), \\
& p(C)=\frac{1}{2}(1-x-y+z) .
\end{aligned}
$$

We may decide that, in a fair game agreement, each member has its initial power multiplied by the same number $k>1$ with

$$
\tilde{P}(A)=p(A) P=k P(A)
$$

and so on for the other voters. Solving for $x, y, z$, we get

$$
\begin{aligned}
& x=1-k \frac{P(B)+P(C)}{P}, \\
& y=1-k \frac{P(A)+P(C)}{P}, \\
& z=1-k \frac{P(A)+P(B)}{P} .
\end{aligned}
$$

If we suppose that $P(A)>P(B)>P(C)$, we see that the largest possible value of $k$, denoted by $k_{\max }$, will be reached when $z=0$ and will read

$$
k_{\max }=\frac{P}{P(A)+P(B)} .
$$

\footnotetext{
${ }^{9}$ In the usual interpretation, a new possibility occurs. There is a non zero probability than $A$ voting 'yes' and the global voting being 'no', a change of opinion of $A$ brings a new random drawing of the global situation with a possible answer 'yes'. Such situations must be interpreted as a negative power and brings agreement with the correlation interpretation.
} 


\begin{tabular}{c|cccccccccccccccc}
$\operatorname{voter} A$ & $\mathrm{Y}$ & $\mathrm{Y}$ & $\mathrm{Y}$ & $\mathrm{Y}$ & $\mathrm{Y}$ & $\mathrm{Y}$ & $\mathrm{Y}$ & $\mathrm{Y}$ & $\mathrm{N}$ & $\mathrm{N}$ & $\mathrm{N}$ & $\mathrm{N}$ & $\mathrm{N}$ & $\mathrm{N}$ & $\mathrm{N}$ & $\mathrm{N}$ \\
$\operatorname{voter} B$ & $\mathrm{Y}$ & $\mathrm{Y}$ & $\mathrm{Y}$ & $\mathrm{Y}$ & $\mathrm{N}$ & $\mathrm{N}$ & $\mathrm{N}$ & $\mathrm{N}$ & $\mathrm{Y}$ & $\mathrm{Y}$ & $\mathrm{Y}$ & $\mathrm{Y}$ & $\mathrm{N}$ & $\mathrm{N}$ & $\mathrm{N}$ & $\mathrm{N}$ \\
$\operatorname{voter} C$ & $\mathrm{Y}$ & $\mathrm{Y}$ & $\mathrm{N}$ & $\mathrm{N}$ & $\mathrm{Y}$ & $\mathrm{Y}$ & $\mathrm{N}$ & $\mathrm{N}$ & $\mathrm{Y}$ & $\mathrm{Y}$ & $\mathrm{N}$ & $\mathrm{N}$ & $\mathrm{Y}$ & $\mathrm{Y}$ & $\mathrm{N}$ & $\mathrm{N}$ \\
$\operatorname{voter} D$ & $\mathrm{Y}$ & $\mathrm{N}$ & $\mathrm{Y}$ & $\mathrm{N}$ & $\mathrm{Y}$ & $\mathrm{N}$ & $\mathrm{Y}$ & $\mathrm{N}$ & $\mathrm{Y}$ & $\mathrm{N}$ & $\mathrm{Y}$ & $\mathrm{N}$ & $\mathrm{Y}$ & $\mathrm{N}$ & $\mathrm{Y}$ & $\mathrm{N}$ \\
\hline $\operatorname{Pr}\left(\right.$ 'yes') $^{\prime}$ & 1 & $1-\delta$ & $1-\gamma$ & $\lambda$ & $1-\beta$ & $\mu$ & $\nu$ & $\alpha$ & $1-\alpha$ & $1-\nu$ & $1-\mu$ & $\beta$ & $1-\lambda$ & $\gamma$ & $\delta$ & 0
\end{tabular}

Table 8: Four member alliance: probability of a 'yes' vote in the random game .

Consequently a sufficient and necessary condition for a three member alliance is

$$
P>P(A)+P(B)
$$

Notice that with $x=y=z=0$, we recover $p(A)=p(B)=p(C)=1 / 2$ i.e. a set of values given by the voting game. Since in the random game $x, y, z$ are arbitrary, the possibility of a three member alliance is extended and the usual voting game is embedded in the random game.

We finally consider a four member alliance of voters $A, B, C$ and $D$, with powers such that $P(A)>P(B)>P(C)>P(D)$. The alliance power is $P$. The decision scheme is given in Table 8 where $\alpha, \beta, \gamma, \delta$ are the probabilities to follow the opinion of respectively $A, B, C, D$ when the three others have an opposite opinion; $\lambda$ is the probability to follow the opinion of the couple $A B$ against the opinion of $C$ and $D ; \mu$ is the probability to follow the opinion of $A$ and $C$ against $B$ and $D ; \nu$ is the probability to follow the opinion of $A$ and $D$ against $B$ and $C$. All these data are summarized in Table 8. Thus, the four power indices inside the alliance are obtained by the correlation method and read

$$
\begin{aligned}
& p(A)=\frac{1}{4}(\alpha-\beta-\gamma-\delta+\lambda+\mu+\nu), \\
& p(B)=\frac{1}{4}(2-\alpha+\beta-\gamma-\delta+\lambda-\mu-\nu), \\
& p(C)=\frac{1}{4}(2-\alpha-\beta+\gamma-\delta-\lambda+\mu-\nu), \\
& p(D)=\frac{1}{4}(2-\alpha-\beta-\gamma+\delta-\lambda-\mu+\nu) .
\end{aligned}
$$

We immediately see that

$$
p(A)+p(B)+p(C)+p(D)=\frac{1}{2}(3-\alpha-\beta-\gamma-\delta) .
$$

So, in a first step, we take $\alpha=\beta=\gamma=\delta=0$ which means that, in a split $3 / 1$, we follow the opinion of the majority. Supposing now that, thanks to the alliance, each member has its power multiplied by $k$, we compute $\lambda, \mu, \nu$ and $k$ and get

$$
\lambda=\frac{2 P(A)+2 P(B)-P(C)-P(D)}{P(A)+P(B)+P(C)+P(D)}
$$




$$
\begin{aligned}
\mu & =\frac{2 P(A)+2 P(C)-P(B)-P(D)}{P(A)+P(B)+P(C)+P(D)} \\
\nu & =\frac{2 P(A)+2 P(D)-P(B)-P(C)}{P(A)+P(B)+P(C)+P(D)} \\
k & =\frac{3}{2} \frac{P}{P(A)+P(B)+P(C)+P(D)}
\end{aligned}
$$

$\lambda, \mu, \nu$ being probabilities, the conditions $0<\lambda$ (resp. $\mu, \nu)<1$ bring relations between $P(A)$, $P(B), P(C), P(D)$. In fact, we have $\lambda>\mu>\nu>0($ using $P(A)>P(B)>P(C)>P(D)$ ). Now from equations (32) and (34), $\lambda<1$ brings the new relation

$$
P(A)+P(B)<2[P(C)+P(D)]
$$

Equality between $P(A), P(B), P(C), P(D)$ gives $\lambda=\mu=\nu=1 / 2$ and consequently $p(A)=p(B)=p(C)=p(D)=3 / 8$. A voting game is possible in this case with one vote to each player and a quota of 2.5 or 1.5 to restore the symmetry, as discussed above.

Keeping $P(B)=P(C)=P(D)$ but increasing $P(A)$, we notice that the three configurations, $A B$ versus $C D, A C$ versus $B D$ and $A D$ versus $B C$ represent identical ratio of power. We must consequently keep $\lambda=\mu=\nu$ and increase these last quantities. At the value 1 , we get $p(B)=p(C)=p(D)=1 / 4$ and $p(A)=3 / 4$. This allows a situation where the power of $A$ is three times the power of the others. For values bigger than three, and, more generally when equation (36) is not fulfilled, a new solution must be found.

First we designate by $\mathcal{A}, \mathcal{B}, \mathcal{C}$ and $\mathcal{D}$, the ratios $P(A) / P, P(B) / P, P(C) / P$ and $P(D) / P$. The new situation (inversion of inequality (35) indicates strong power for $A$ alone or $A$ and $B$ ) emerges with the value $\lambda=1$ which will be kept. Since $p(A)=k \mathcal{A}, p(B)=k \mathcal{B}$, .., equations (27)-(30) are written

$$
\begin{aligned}
4 k \mathcal{A} & =\alpha-\beta-\gamma-\delta+\lambda+\mu+\nu \\
4 k \mathcal{B} & =2-\alpha+\beta-\gamma-\delta+\lambda-\mu-\nu \\
4 k \mathcal{C} & =2-\alpha-\beta+\gamma-\delta-\lambda+\mu-\nu \\
4 k \mathcal{D} & =2-\alpha-\beta-\gamma+\delta-\lambda-\mu+\nu
\end{aligned}
$$

Adding (37) and (38) with $\lambda=1$, we get

$$
4 k(\mathcal{A}+\mathcal{B})=4-2 \gamma-2 \delta
$$

Because inequality (36) is no longer fulfilled, we cannot keep $\alpha=\beta=\gamma=\delta=0$; logic imposes that we first take $\alpha$ (and may be $\beta$ ) different from 0 . Then to maximize $k$ equation (41) gives

$$
k=\frac{1}{\mathcal{A}+\mathcal{B}}
$$


and the $\gamma=\delta=0$. We notice also that a value of $\lambda<1$ would decrease $k$ justifying the intuitive choice $\lambda=1$. Now, since we have four equations and five unknowns $(\alpha, \beta, \mu, \nu, k)$ we can keep one (except $k$ ) arbitrary. We take $\beta$ and obtain

$$
\begin{aligned}
\alpha & =\frac{\mathcal{A}+\mathcal{B}-2(\mathcal{C}+\mathcal{D})}{\mathcal{A}+\mathcal{B}}-\beta \\
\mu & =\frac{\mathcal{A}+2 \mathcal{C}-\mathcal{B}}{\mathcal{A}+\mathcal{B}}+\beta \\
\nu & =\frac{\mathcal{A}+2 \mathcal{D}-\mathcal{B}}{\mathcal{A}+\mathcal{B}}+\beta \\
k & =\frac{1}{\mathcal{A}+\mathcal{B}}
\end{aligned}
$$

If in equations (42)-(44) we take $\beta=0$, we get

$$
1>\mu>\nu>0
$$

and

$$
1>\alpha>0
$$

From a mathematical point of view, the solution is correct and perfectly admissible. But from a logical point of view, it can sometimes appear strange. If $1=\lambda>\mu>\nu$ respect the power hierarchy $A B, A C, A D$, we expect $\nu>\alpha$, i.e. the couple $A D$ should be stronger than $A$ alone. Equations (42) and (44) implies $\mathcal{B}<\mathcal{C}+2 \mathcal{D}$. In that case, taking $\beta=0$ in equations (42)-(44) preserves the hierarchy $A B, A C, A D, A, B, \ldots$ But for $\mathcal{B}>\mathcal{C}+2 \mathcal{D}$ it is necessary to take $\beta \neq 0$. Keeping $\alpha=\nu$ gives

$$
\begin{aligned}
\beta & =\frac{\mathcal{B}-\mathcal{C}-2 \mathcal{D}}{\mathcal{A}+\mathcal{B}} \\
\alpha & =\frac{\mathcal{A}-\mathcal{C}}{\mathcal{A}+\mathcal{B}} \\
\lambda & =1 \\
\mu & =\frac{\mathcal{A}+\mathcal{C}-2 \mathcal{D}}{\mathcal{A}+\mathcal{B}} \\
\nu & =\frac{\mathcal{A}-\mathcal{C}}{\mathcal{A}+\mathcal{B}}
\end{aligned}
$$

with preservation of the hierarchy.

The case $\alpha=\nu$ is quite logical if $\mathcal{D}=0$ (see below the discussion of this liming case and the connection with the three players game). For $\mathcal{D} \neq 0$, we may increase the value of $\beta$ given by equation (46) and select

$$
\beta>(\mathcal{B}-\mathcal{C}-2 \mathcal{D}) /(\mathcal{A}+\mathcal{B})
$$




\begin{tabular}{|c|c|c|c|c|c|c|c|}
\hline & $\mathcal{A}+\mathcal{B}<2(\mathcal{C}+\mathcal{D})$ & $\mathcal{A}+\mathcal{B}=2(\mathcal{C}+\mathcal{D})$ & \multicolumn{5}{|c|}{$\mathcal{A}+\mathcal{B}>2(\mathcal{C}+\mathcal{D})$} \\
\hline & \multicolumn{4}{|c|}{$\mathcal{B}<\mathcal{C}+2 \mathcal{D}$} & $\mathcal{B}=\mathcal{C}+2 \mathcal{D}$ & \multicolumn{2}{|c|}{$\mathcal{B}>\mathcal{C}+2 \mathcal{D}$} \\
\hline $\mathcal{A}$ & 6 & 7 & 8 & 9 & 10 & 11 & 12 \\
\hline $\mathcal{B}$ & 6 & 7 & 8 & 9 & 10 & 11 & 12 \\
\hline $\mathcal{C}$ & 4 & 4 & 4 & 4 & 4 & 4 & 4 \\
\hline $\mathcal{D}$ & 3 & 3 & 3 & 3 & 3 & 3 & 3 \\
\hline$\alpha$ & 0 & 0 & $1 / 8 \quad 1 / 16$ & $2 / 9 \quad 1 / 9$ & $3 / 10 \quad 3 / 20$ & $7 / 22 \quad 4 / 22$ & $1 / 3 \quad 5 / 24$ \\
\hline$\beta$ & 0 & 0 & $0 \quad 1 / 16$ & $\begin{array}{ll}0 & 1 / 9\end{array}$ & $3 / 20$ & $1 / 22 \quad 4 / 22$ & $1 / 12 \quad 5 / 24$ \\
\hline$\lambda$ & $17 / 19$ & 1 & 1 & 1 & 1 & 1 & 1 \\
\hline$\mu$ & $11 / 19$ & $4 / 7$ & $1 / 29 / 16$ & $4 / 95 / 9$ & $2 / 5 \quad 11 / 20$ & $9 / 22 \quad 12 / 22$ & $5 / 12 \quad 13 / 24$ \\
\hline$\nu$ & $8 / 19$ & $3 / 7$ & $3 / 87 / 16$ & $3 / 9 \quad 4 / 9$ & $3 / 10 \quad 9 / 20$ & $7 / 22 \quad 10 / 22$ & $1 / 3 \quad 11 / 24$ \\
\hline
\end{tabular}

Table 9: Four voter alliance : values of $\alpha, \beta, \lambda, \mu, \nu$ for different values of the power of voters $A, B, C, D$, but with equality $\mathcal{A}=\mathcal{B}$. When two solutions are given, the second one preserves the equality of the power of $A$ and $B$.

but, then, we must check that $\alpha>\beta$. This implies

$$
\beta<\frac{\mathcal{A}+\mathcal{B}-2(\mathcal{C}+\mathcal{D})}{2(\mathcal{A}+\mathcal{B})} \text {. }
$$

Consequently, we must select

$$
\frac{\mathcal{A}+\mathcal{B}-2(\mathcal{C}+\mathcal{D})}{2(\mathcal{A}+\mathcal{B})}>\beta>\frac{\mathcal{B}-\mathcal{C}-2 \mathcal{D}}{\mathcal{A}+\mathcal{B}}
$$

This freedom on $\beta$ can also be used to preserve other properties, for example the symmetry in the coefficients brought by the equality $\mathcal{A}=\mathcal{B}$. A numerical example will clearly illustrate this point. Suppose we have $\mathcal{C}=4, \mathcal{D}=3$ and $\mathcal{A}=\mathcal{B}$ varying from 6 to 12. Table 9 gives two possible results, the first with $\beta=0$, or if $\mathcal{B}>\mathcal{C}+2 \mathcal{D}$, a $\beta$ given by (46), the second with $\beta=\alpha$.

Notice that the symmetrization $\alpha=\beta$ brings $\mu=1-\nu$, a consequence of the equality of power between $A C$ (probability $\mu$ ) and $B C$ (probability $1-\nu$ ). The symmetric solution is more logical.

To summarize we see that we can consider three cases :

1. $\mathcal{A}+\mathcal{B}<2(\mathcal{C}+\mathcal{D})$. Then $\alpha=\beta=\gamma=\delta=0$ and $\lambda, \mu, \nu$ are given by $(32)-(34)$.

2. $\mathcal{A}+\mathcal{B}>2(\mathcal{C}+\mathcal{D})$ and $\mathcal{B}<\mathcal{C}+2 \mathcal{D}$. Then $\beta=\gamma=\delta=0, \lambda=1$, but $\alpha \neq 0$. The values of $\alpha, \mu, \nu$ are given by (42)-(44). In some cases, to preserve symmetry properties, we can also take $\beta \neq 0$ as discussed table 9 .

3. $\mathcal{A}+\mathcal{B}>2(\mathcal{C}+\mathcal{D})$ and $\mathcal{B}>\mathcal{C}+2 \mathcal{D}$. Then $\gamma=\delta=0, \lambda=1$. The values of $\alpha, \mu, \nu$ are still given by (42)-(44) but $\beta$ is somewhat arbitrary. Logical considerations on the hierarchy of combinations brings a choice as given by (51). Further considerations $(\mathcal{D}=0, \mathcal{A}=\mathcal{B})$ may precise the value of $\beta$ and in fact give respectively its lower and upper limit). 
A deeper understanding of the results given by equations (42)-(44) is provided by letting $\mathcal{D}$ go to 0 . Intuitively we expect $D$ to be a dummy and consequently we should recover the results of the three players game. In that case, beside the trivial situation where everybody agree, we have three possibilities $A$ against $B C, B$ against $A C$ and $C$ against $A B$. In the four players game, the first situation corresponds to the union of $A$ against $B C D$ (probability $\alpha$ ) and $A D$ against $B C$ (probability $\nu$ ). These two situations being equally likely the probability is

$$
\frac{\alpha+\nu}{2}=\frac{\mathcal{A}-\mathcal{C}}{\mathcal{A}+\mathcal{B}}
$$

In the same way, the situation corresponding to $B$ against $A C$ is the union of $B$ against $A C D$ (probability $\beta$ ) and $B D$ against $A C$ (probability $1-\mu$ ) for a total of

$$
\frac{\beta+1-\nu}{2}=\frac{\mathcal{B}-\mathcal{C}}{\mathcal{A}+\mathcal{B}}
$$

Last, the situation of $C$ against $A B$ is the union of $C$ against $A B D$ (probability $\gamma=0$ ) and $C D$ against $A B$ (probability $1-\lambda=0$ ) and a total probability of 0 . These three values are indeed the $x, y, z$ of the three players random game formula (22)-(24). Notice that, since $D$ has no power; it is logical to take $\nu$ (probability of following the opinions of $A D$ against $B C$ ) equal to $\alpha$ (probability of following the opinion of $A$ against $B C D$ ). Moreover, with $\mathcal{D}=0$, we are automatically in the case $\mathcal{A}+\mathcal{B}>2(\mathcal{C}+\mathcal{D})$ and $\mathcal{B}>\mathcal{C}+2 \mathcal{D}$, and the use of equations (46)-(50) is legitimated. For five players, or more, new features appear and the results will be given in a forthcoming paper.

Let us now illustrate the difference between the voting and the random games for some possible alliances in the Council of Ministers of the European Union. We consider first a hypothetical alliance formed by Germany $(A)$, Holland $(B)$ and Luxembourg $(C)$, and called by Felsenthal and Machover [3] the Deunelux. In the 15 member EU, considered in [3], we have $P(A)=0.1129$, $P(B)=0.0594, P(C)=0.0229$ and $P=0.1975$. Felsenthal and Machover show (and it is easily checked) that no voting game exists leading to a feasible alliance. Now, consider a three player random game such that $x=0.522, y=0.212$ and $z=0$. We then obtain $\tilde{P}(A)=0.1294$, $\tilde{P}(B)=0.0681$ and $\tilde{P}(C)=0.0262$ : With this random game, the alliance becomes possible since the power of each member is multiplied by 1.15. In the EU27, a similar conclusion holds: the Deunelux alliance is not feasible under any voting game but is possible if a random game is adopted: Taking $x=0.551, y=0.233$ and $z=0$ implies a multiplication by 1.13 of the voting power of each member in the alliance.

Another alliance analyzed in [3] is the Medbloc (Italy, Spain, Portugal, Greece). For the Medbloc, Felsenthal and Machover [3] propose two voting games. The first one is $(4 ; 2,2,1,1)$, that is to say two votes for Italy and Spain, one vote for Portugal and Greece and a quota $Q=4$. This gives, inside the alliance, a power of $1 / 2$ for Italy and Spain and a power of $1 / 4$ for Portugal and Greece. Although the four countries gain voting power thanks to this alliance in the EU15, Italy and (particularly) Spain get the lion's share: Their final power $\tilde{P}$ increases by $57 \%$ and $90 \%$ 
(repectively), while Portugal and Greece obtain a lower increase of $49 \%$. These two countries will certainly prefer the second voting game $(3 ; 1,1,1,1)$, with for each country in the alliance a power $p=3 / 8$. With this voting game, the final power of Portugal and Greece increases by $124 \%$ (18\% for Italy and $42 \%$ for Spain). By contrast, the solution with the random game provides to each country the same proportional increase (about 72\%). ${ }^{10} 11$

\section{Conclusion}

In this paper, we point out four (more or less) new ideas.

1. The interest of an alliance is not so much founded on affinity of opinions but on a mechanical "scale effect" with a needed game inside the alliance.

2. In the so-called asymptotic limit, interesting laws emerge : Power proportional to the number of votes, power of an alliance equal to the sum of member's power; with in addition, an amusing benefit : This limit makes easier the explanation of the power concept to the general public.

3. In voting process, as in many other problems in game theory, and also in some computational techniques, the introduction of randomness is not only useful but may be unavoidable in some problems. This idea is certainly not easy to pass to the politician community.

4. In EU27, the number of countries is now sufficiently large (and the weights sufficiently scattered) so that the Gaussian approximation becomes valid in most institutional scenarios.

Acknowledgment. We gratefully acknowledge the financial support of the French ministry of research through the programme ACI "Terrains, Techniques, Théories: Travail Interdisciplinaire en Sciences Humaines et Sociales". We also thank the participants of the VPP workshop held in Caen on July 3-5 2003 for their comments on a preliminary version of this paper. The remarks of two anonymous referees have proved to be fruitful to improve this paper.

\footnotetext{
${ }^{10}$ It is interesting to point out that Felsenthal and Machover suggest a coin tossing to decide between the two games which is another step toward the introduction of randomness in the problem.

${ }^{11}$ Notice that in the EU27, the random game is not really necessary: The voting game $(4 ; 2,2,1,1)$ can be considered as satisfactory. After computation, it turns out that, under this voting game, the final voting power of each member in the alliance increases approximatively by the same proportion: $78 \%$ for Italy, $87 \%$ for Spain, $86 \%$ for Portugal and $86 \%$ for Greece. With a random game suitably chosen, the power increase is about $83 \%$ for each country.
} 


\section{References}

[1] Banzhaf, J. F. (III) : Weighted Voting Doesn't Work, Rutgers Law Review 19 Winter, pp 317-343, 1965.

[2] Felsenthal D.S., Machover M. : The Measurement of Voting Power: Theory and Practice, Problems and Paradoxes, Edward Elgar Publishers, London, 1998.

[3] Felsenthal D.S., Machover M. : Annexations and Alliances: When Are Blocs Advantageous a Priori?, Social Choice and Welfare 19, p 295-312, 2002.

[4] Felsenthal D.S., Machover M. : The Treaty of Nice and Qualified Majority Voting, Social Choice and Welfare 18, p 431-464, 2001.

[5] Fristedt B., Gray L. : A Modern Approach to Probability Theory, Birkhauser Publishers, Boston, 1996.

[6] Huang, K. : Statistical Mecanics, John Wiley \& Sons Corporated Publisher, New-York, 1963.

[7] Kittel C., Elementary Statistical Physics, Krieger Publishing Company; Reprint edition, 1988.

[8] Leech D. : Computing Power Indices for Large Voting Games, Management Sciences 49, p 831-837, 2003.

[9] Lindner I., Machover M. : LS Penrose's Limit Theorem: Proof of some Special Cases, Mathematical Social Sciences 47, p 37-49, 2004.

[10] Owen G. : Multilinear Extensions of Games. Management Sciences 18, p 64-79, 1972.

[11] Owen G. : Multilinear Extensions and the Banzhaf Value, Naval Research Logistics Quaterly 22, p 741-750, 1975.

[12] Penrose L.S., The Elementary Statistics of Majority Voting, Journal of the Royal Statistical Society 109, p 53-57, 1946.

[13] Penrose L.S., On the Objective Study of Crowd Behavior, H.K. Lewis \& Co, London, 1952.

[14] Rae D., Decision Rules and Individual Values in Constitutional Choice, American Political Science Review 63, p 40-56, 1969.

[15] Tribelsky M.I. : General Solution to the Problem of the Probability Density for Sums of Random Variables, Phys. Rev. Letters 89 7, 2002. 


\section{Appendix I.}

We provide an exhaustive examination of the various distributions of voting power that can be obtained depending on the quota $Q$ for a three players voting game. Let $a, b, c$ be the votes of respectively players $A, B, C$, with $a>b>c$. Two cases must be examined

1. $a<b+c$,

2. $a>b+c$.

Figures 8 and 9 give the seven intervals which can be distinguished in cases $a<b+c$ and $a>b+c$ respectively. For these two cases, tables 10 and 11 gives the power of $A, B, C$ depending of the location of $Q$ (the quota) in the different segments represented figures 8 and 9. Moreover the tables give the probability of a 'yes' answer of the three players alliance.

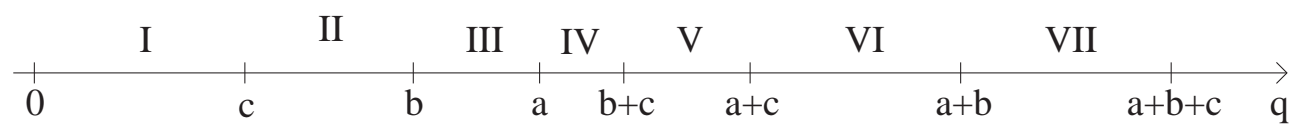

Figure 8: The different interval to consider for the value $Q$ in the case $a<b+c$.

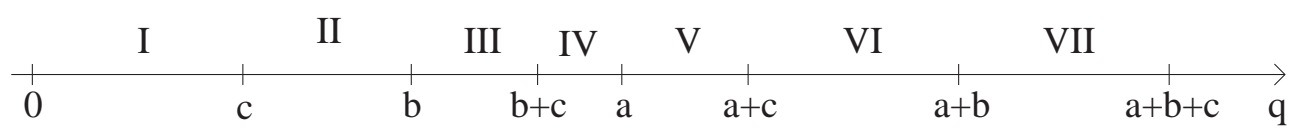

Figure 9: The different interval to consider for the value $Q$ in the case $a>b+c$.

We notice that, for a quota $Q$ and for a quota $T-Q$ (where $T$ is the total number of votes), the powers of each players are identical while the probability of 'yes' and 'no' global answer are switched.

Looking at the two cases, the only difference concerns the powers $P(A), P(B), P(C)$ in the central zone IV.

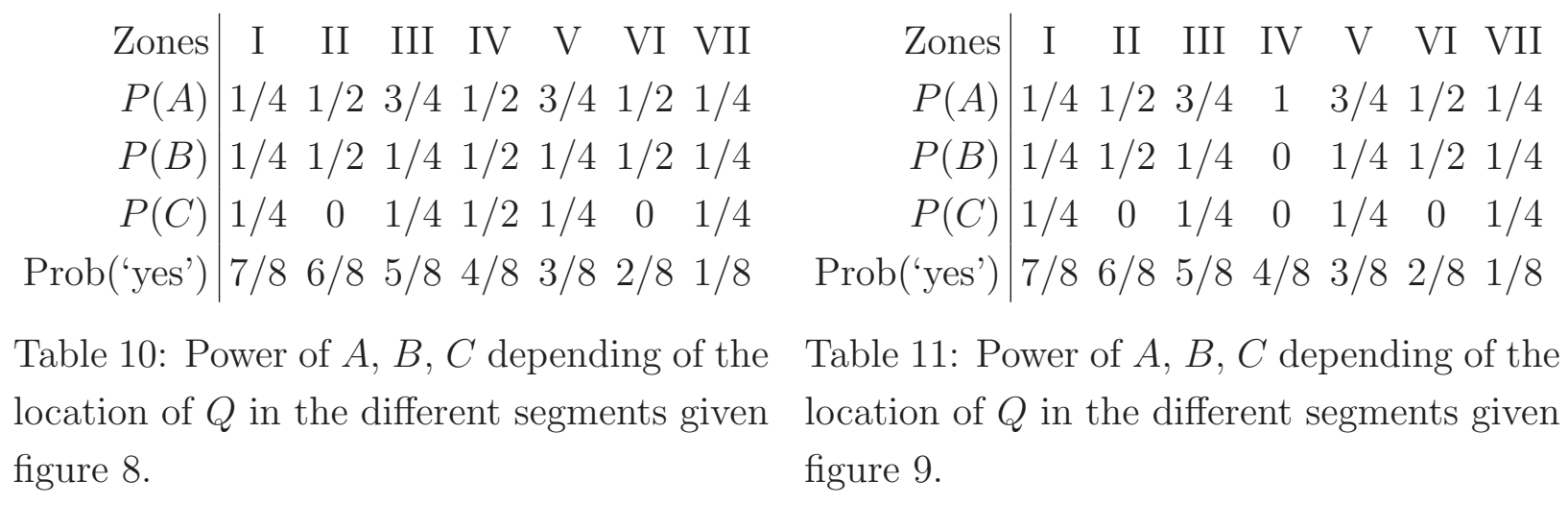

